\title{
Parnassia palustris, das Sumpf-Herzblatt
}

\author{
Armin Jagel
}

\begin{abstract}
The Northern Grass-of-Parnassus (Parnassia palustris) is a protected plant under German law. Its deceptive flowers lurk in pollinators by mimicking the presence of copious nectar and pollen. Morphology, ecology, and pollination biology of the plant are outlined.
\end{abstract}

\section{Zusammenfassung}

Das Sumpfherzblatt (Parnassia palustris) ist in Deutschland eine geschützte Art. Die Blüten täuschen reichlich Nektar und Pollen vor, um Bestäuber anzulocken. Morphologie, Ökologie und Bestäubungsbiologie werden erläutert.
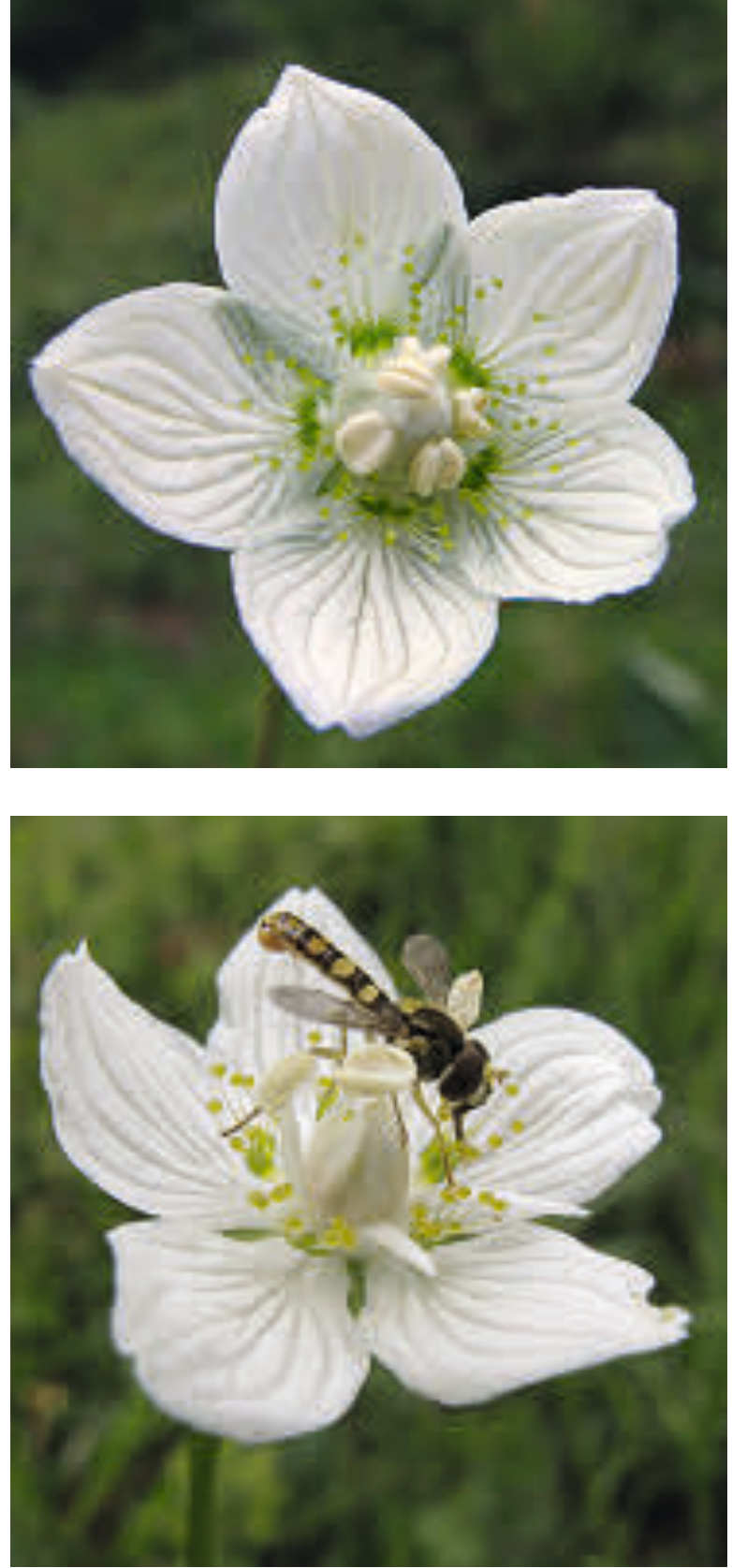

\section{Einleitung}

Das Sumpf-Herzblatt (Parnassia palustris, Celastraceae) erhielt seinen deutschen Namen von seinem bevorzugten Lebensraum auf feuchten, sumpfigen Standorten und seinen oft herzförmigen Blättern. Es ist eine eurasiatisch verbreitete Art, die in den Alpen noch relativ häufig anzutreffen ist. Auf der Nordhalbkugel erstreckt sich das Verbreitungsgebiet von Island über Skandinavien und Japan bis nach Kanada.

In Deutschland ist das Sumpf-Herzblatt außerhalb der Alpen durch Entwässerungsmaßnahmen und Überdüngung stark bedroht und steht unter Naturschutz. In Nordrhein-Westfalen beispielsweise ist die Art eine Seltenheit, wobei die Bestände immer weiter abnehmen (vgl. Haeupler et al. 2003). Noch Ende des 19. Jh. war die Art allerdings in Nordrhein-Westfalen noch weit verbreitet und besiedelte dort ein viel breiteres Spektrum an Standorten, als man es heute glauben mag. Beckhaus (1893) schreibt: „Auf torfigen, besonders eisenhaltigen Sumpf- und Waldwiesen fast überall, auch [...] auf trockenen Triften der Kalkberge, auch des Keupers, nicht selten ..." Neben all den Gefahren, die auch heute noch zum Rückgang der Art

Abb. 1 (oben): Sumpfherzblatt-Blüte am ersten Tag: Die Staubbeutel liegen dem Fruchtknoten an, ein Staubbeutel (links oben) schiebt sich über den Fruchtknoten ins Blütenzentrum und öffnet sich.

Abb. 2 (unten): Schwebfliege untersucht die glitzernden, aber nektarfreien Drüsenköpfchen der Staminodien. 
führen, droht ihnen eine offenbar nicht, denn das Sumpf-Herzblatt wird von keinem Tier gefressen.

Die Blüten wirken auf den ersten Blick unspektakulär. Sie haben aber einige Überraschungen zu bieten, denn bei genauer Betrachtung stellt sich heraus, dass sie ihre Bestäuber betrügen und anderseits durch eine ausgeklügelte Blührhythmik die Fremdbestäubung fördern. Einer Blüte kann sogar angesehen werden, wie alt sie ist und wie viele Tage sie noch blühen wird.

\section{Systematik}

Die Gattung umfasst etwa 70 Arten (MabberLEy 2008), in Europa kommt aber nur Parnassia palustris vor. Die Systematik von Parnassia ist unbefriedigend geklärt. Die Merkmale der Gattung sind so einzigartig, dass sie oft in die eigene Familie der Parnassiaceae gestellt wird. Von früheren Autoren wurde Parnassia den Steinbrechgewächsen (Saxifragaceae) oder den Sonnentaugewächsen (Droseraceae) zugeordnet (vgl. Hegr 1923). Die moderne Systematik, die auf molekularen Daten basiert, führte aber dazu, Parnassia zu den Celastraceae (Spindelbaumgewächse) zu stellen. Aus morphologischer Sicht ist dies jedoch schwer nachvollziehbar.

\section{Trickreiche Blütenbiologie}

Die Blüten öffnen sich ungewöhnlich spät im Jahr, oft erst im August und dann bis in den Oktober hinein. Ihr Aufbau ist in großen Teilen für zweikeimblättrige Arten auf den ersten Blick nicht ungewöhnlich: Auf fünf grüne Kelchblätter folgen fünf weiße Kronblätter, die

Abb. 3 (oben): Ein Blüte am zweiten Tag. Das Staubgefäß des ersten Tages hat sich nach außen gebogen (rechts unten), ein zweites Staubblatt (rechts oben) schiebt sich ins Zentrum der Blüte.

Abb. 4 (Mitte): Blüte am sechsten Tag von der Seite: Die Blüte ist im weiblichen Zustand, die Narbe ist geöffnet und jetzt erst empfangsbereit.

Abb. 5 (unten): Seitenansicht einer Blüte. Staminodium mit an der Spitze ausgebildeten glänzenden „Drüsenköpfchen“.
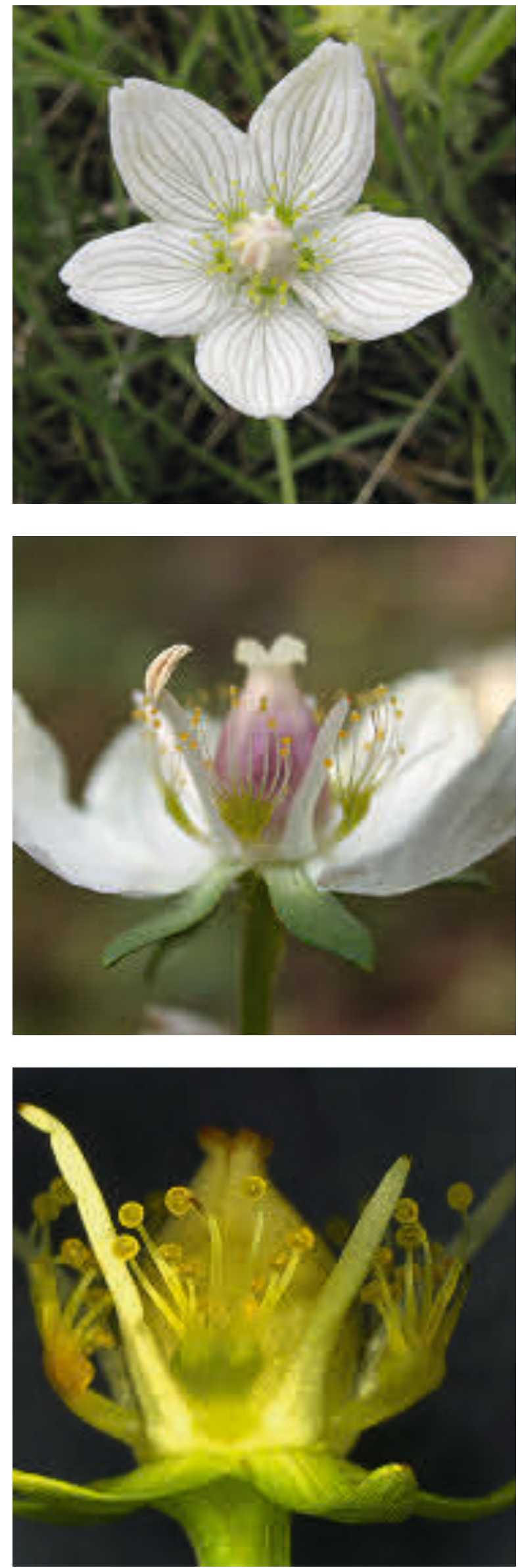

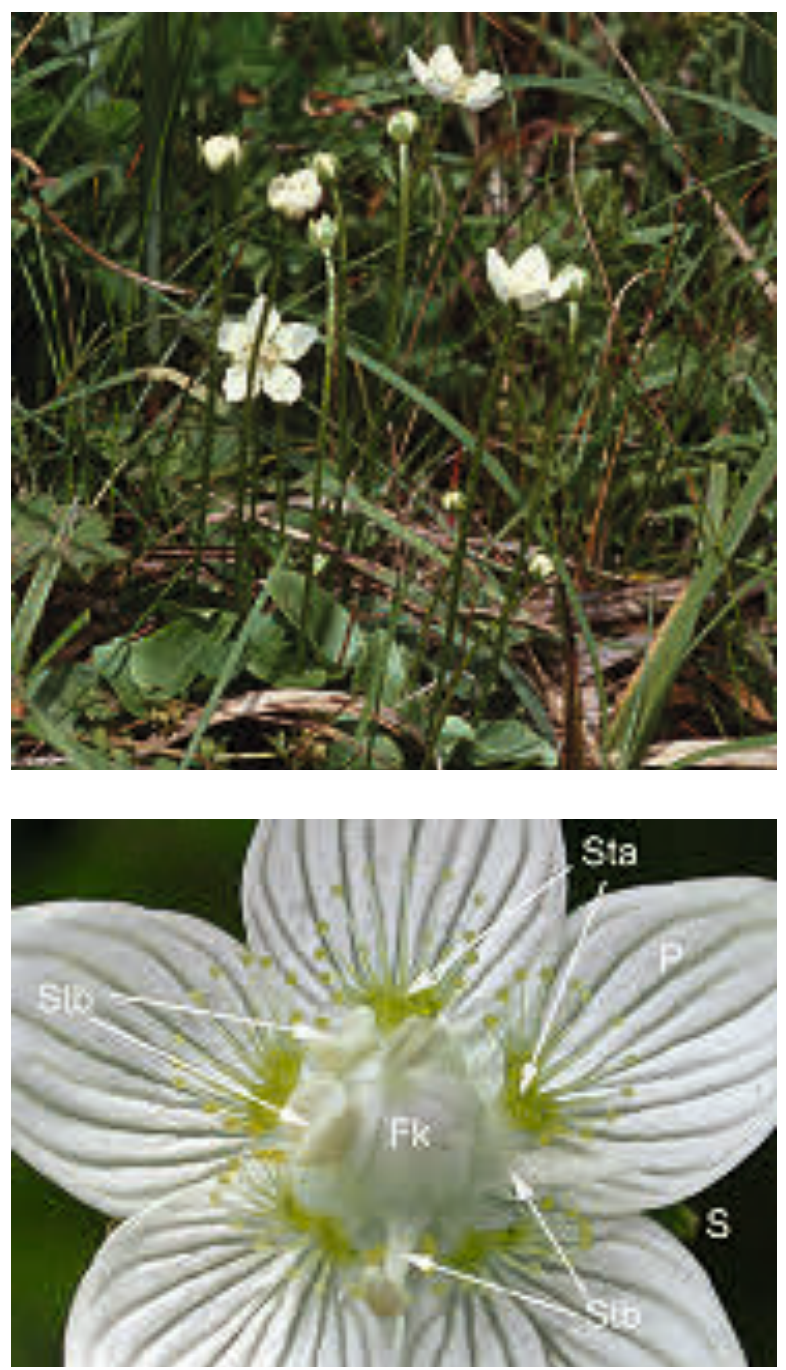

mit dunklen strichförmigen Saftmalen versehen sind. Es schließt sich ein Kreis aus fünf normal gebauten Staubbeuteln an. Dann aber folgt nach innen ein weiterer Kreis, der aus ungewöhnlich gestalteten Staubblättern aufgebaut ist. Diese sind so stark abgewandelt, dass sie nicht mehr wie Staubblätter aussehen und auch ihre ursprüngliche Funktion (die Bildung von Pollen) verloren haben. Sie sind steril und werden Staminodien genannt. Beim Sumpf-Herzblatt bilden die Staminodien an der Basis wenig, dafür aber duftenden Nektar, um Bestäuber anzulocken. Am oberen Ende der Staminodien befinden sich dagegen mehrere lang gestielte Kügelchen, die zwar glänzenden Tropfen ähneln, aber trocken sind und keinen Nektar abgeben. Diese Kügelchen werden in der Literatur als Stieldrüsen bezeichnet, sind aber keine Drüsen, da sie kein Sekret produzieren. Die
Blüten täuschen durch sie viel mehr Nektar vor, als sie in Wirklichkeit zu bieten haben. Durch die Staminodien wird auch mehr Pollen vorgetäuscht, als wirklich im Angebot ist (Düll \& Kutzelnigg 2010). Auf die Nektarattrappen fallen besonders Fliegen herein, weshalb die Blüten als Fliegentäuschblumen bezeichnet werden. Die Fliegen gelangen auf der Suche nach dem Nektar mehrfach in das Zentrum der Blüte und kommen dabei in jungen Blüten mit den geöffneten Staubbeuteln und im letzten (weiblichen) Stadium mit der fertilen Narbe in Berührung. Dabei werden die Blüten bestäubt. Auch Ameisen besuchen häufig auf der Suche nach Nektar Parnassia-Blüten. Eine Bestäubung der Blüte erfolgt in diesem Fall aber wohl nicht.

Die Blüten des Sumpf-Herzblattes lassen erkennen, wie alt sie sind: Sie sind vormännlich (= protandrisch), d. h. die Staubblätter einer Blüte reifen vor dem Fruchtknoten und den Narben. Die fünf fruchtbaren, zunächst noch geschlossenen Staubbeutel liegen anfangs dem zentral stehenden Fruchtknoten an. Am ersten Tag der Blütezeit streckt sich eines der Staubblätter über den Fruchtknoten ins Zentrum der Blüte. Die Staubbeutel nur dieses einen Staubblattes öffnen sich nun nach oben und präsentieren ihren Pollen, der dem Bestäuber am Bauch aufgedrückt wird. Am nächsten Tag hat sich das Stielchen (Filament) des Staubblattes nach außen gedreht und die Staubbeutel abgeworfen. Nun streckt sich ein zweites Staubblatt ins Blütenzentrum und öffnet sich. Entsprechend geht es weiter, bis sich auch das fünfte Staubblatt geöffnet und aus dem Blütenzentrum wieder entfernt hat. Da die Bewegung des Staubblattes und das Öffnen der Pollen-

Abb. 6 (oben): Blühende Pflanze auf einer Wiese in der Hohen Tatra (Polen, 2001).

Abb. 7 (unten): Aufbau der Blüte: Fk = Fruchtknoten, $\mathrm{P}=$ Kronblatt (Petalum), $\mathrm{S}=$ Kelchblatt (Sepalum), Sta $=$ Staminodium, Stb $=$ Staubbeutel.

Abb. 8 (Seite 137): Sumpf-Herzblatt in einem Gletschervorfeld im Lötschental zusammen mit Fleischers Weidenröschen. 


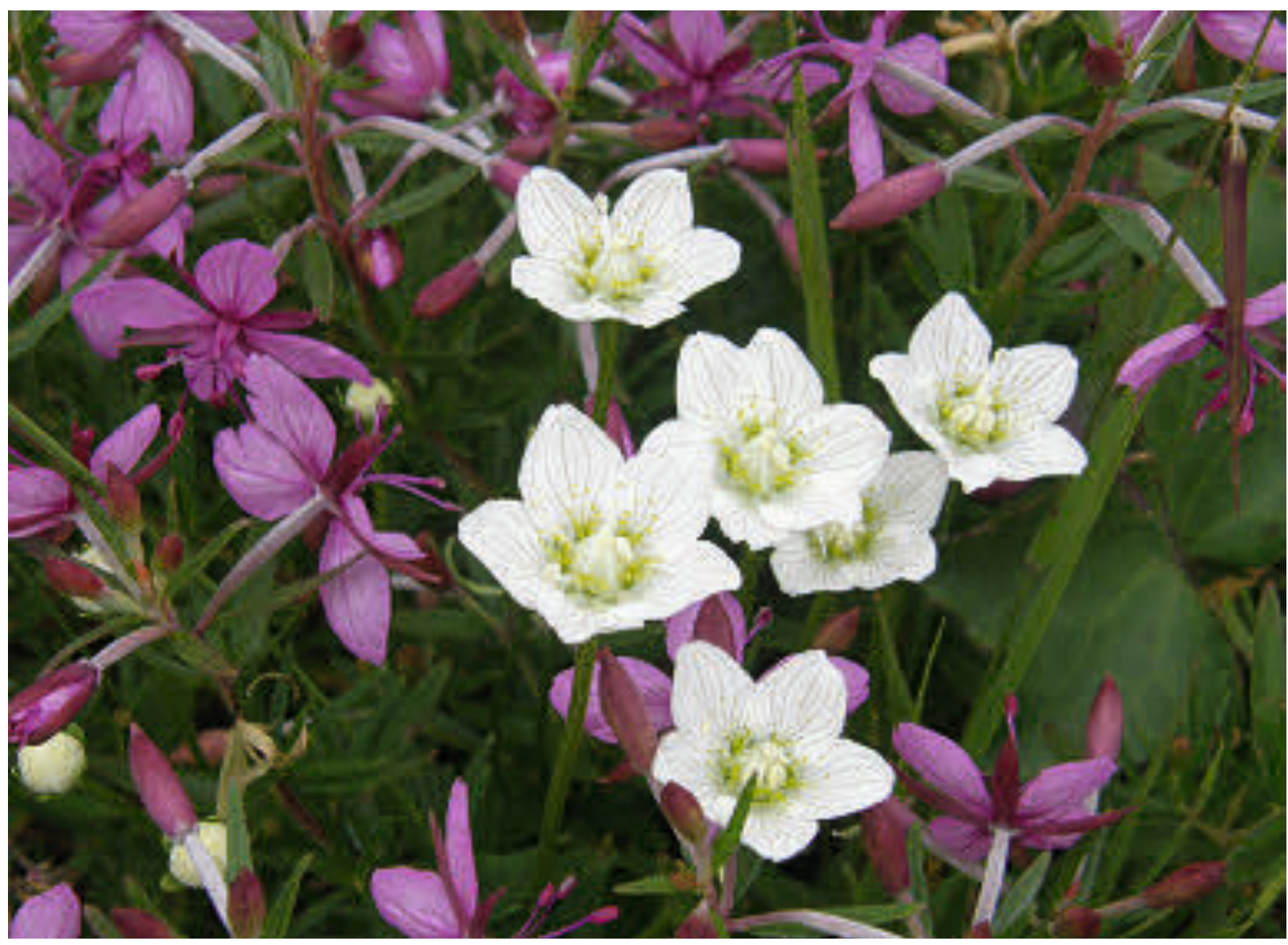

säcke in der Regel etwa einen Tag andauern, kann so auf das Alter einer Blüte geschlossen werden (Hess 1990).

Am sechsten Tag liegt nun der Fruchtknoten im Zentrum der Blüte frei und die bis zu diesem Zeitpunkt geschlossene Narbe öffnet sich, womit die Blüte in den weiblichen Zustand übergeht. Pollen der eigenen Blüte ist nicht mehr vorhanden, die Narbe kann nur noch fremdbestäubt werden. Dieser ausgeklügelte und präzise Mechanismus bei der Blüte des Sumpf-Herzblattes gewährleistet, dass der Pollen genau an der Position präsentiert wird, wo bei einer anderen, bereits im weiblichen $\mathrm{Zu}$ stand befindlichen Blüte, die empfängnisbereite Blütennarbe liegt.

\section{Literatur}

Becknaus, K. 1893: Flora von Westfalen. Die in der Provinz von Westfalen wild wachsenden Gefäßpflanzen. - Münster (Reprint 1993).

Düll R. \& Kutzelnigg, H. 2011: Taschenlexikon der Pflanzen Deutschlands und angrenzender Länder. Wiebelsheim.

Hegi, G. 1923: Illustrierte Flora von Mitteleuropa, Bd. 4 (2). - München.

Hess, D. 1990: Die Blüte. - Stuttgart.

Haeupler, H., Jagel, A. \& Schumacher, W. 2003 :

Verbreitungsatlas der Farn- und Blütenpflanzen in Nordrhein-Westfalen. - Recklinghausen: LÖBF. Mabberley, D. J. 2008: Mabberley's plant book, 3. Aufl. - Cambridge.

Raabe, U., Wolf-Straub, R., Büscher, D., Diekjobst, H., Fasel, P., Foerster, E., Götte, R., Jagel, A., Kaplan, K., Koslowski, I., KutzelnigG, H., Schumacher, W. \& Vanberg, C. 2011: Rote Liste und Artenverzeichnis der Farn- und Blütenpflanzen Pteridophyta et Spermatophyta - in Nordrhein-Westfalen. - Recklinghausen. 